\title{
A Sensitive Method Approach for Chromatographic Analysis of Gas Streams in Separation Processes Based on Columns Packed with an Adsorbent Material
}

\author{
I. A. A. C. Esteves, ${ }^{1}$ G. M. R. P. L. Sousa, ${ }^{2}$ R. J. S. Silva, ${ }^{3}$ R. P. P. L. Ribeiro, ${ }^{1}$ \\ M. F. J. Eusébio, ${ }^{1}$ and J. P. B. Mota ${ }^{1}$ \\ ${ }^{1}$ LAQV, REQUIMTE, Departamento de Química, Faculdade de Ciências e Tecnologia, Universidade Nova de Lisboa, \\ 2829-516 Caparica, Portugal \\ ${ }^{2}$ Saudi Basic Industries Corporation (SABIC), P.O. Box 5101, Riyadh 11422, Saudi Arabia \\ ${ }^{3}$ Instituto de Biologia Experimental e Tecnológica (iBET), Apartado 12, 2781-901 Oeiras, Portugal \\ Correspondence should be addressed to I. A. A. C. Esteves; iaesteves@fct.unl.pt
}

Received 1 February 2016; Accepted 19 May 2016

Academic Editor: Fernando Lusquiños

Copyright (C) 2016 I. A. A. C. Esteves et al. This is an open access article distributed under the Creative Commons Attribution License, which permits unrestricted use, distribution, and reproduction in any medium, provided the original work is properly cited.

\begin{abstract}
A sensitive method was developed and experimentally validated for the in-line analysis and quantification of gaseous feed and product streams of separation processes under research and development based on column chromatography. The analysis uses a specific mass spectrometry method coupled to engineering processes, such as Pressure Swing Adsorption (PSA) and Simulated Moving Bed (SMB), which are examples of popular continuous separation technologies that can be used in applications such as natural gas and biogas purifications or carbon dioxide sequestration. These processes employ column adsorption equilibria on adsorbent materials, thus requiring real-time gas stream composition quantification. For this assay, an internal standard is assumed and a single-point calibration is used in a simple mixture-specific algorithm. The accuracy of the method was found to be between $0.01 \%$ and $0.25 \%(-\mathrm{mol})$ for mixtures of $\mathrm{CO}_{2}, \mathrm{CH}_{4}$, and $\mathrm{N}_{2}$, tested as case-studies. This makes the method feasible for streams with quality control levels that can be used as a standard monitoring and analyzing procedure.
\end{abstract}

\section{Introduction}

Adsorption separation is a phenomenon in which one or more components of a fluid (adsorbate) are extracted via selective bonding to a solid (adsorbent) medium [1-3]. This is the basis for every adsorption separation process. Periodic adsorption processes, such as single or multibed Pressure Swing Adsorption (PSA), have been extensively developed and applied in industry for gas separation and are energy efficient alternatives to other separation techniques such as cryogenic distillation. Much progress has been achieved in improving their performance with respect to both the process economics and the attainable purity of the products by investigating new process configurations or giving a better reuse to some topical waste gases like biogas produced from biomass [4]. Simulated Moving Bed (SMB) is a continuous adsorptive separation process with various applications, many of which are difficult to handle using other techniques. Recently, the feasibility of SMB operation for gas separation has been addressed and its feasibility demonstrated, with focus on cyclic modulation of the internal flow rates $[5,6]$.

Long gone are the days where the development of SMB and PSA process schemes and their experimental validation were analyzed by manual and discrete sampling quantification chromatographic techniques. Nowadays, for the applications of interest the coupling of the separation/purification process unit with a spectrometry system can be done through a capillary line, in order to bring the required continuous sample streams into the analyzer at the specified pressure, while maintaining the high vacuum necessary for proper spectrometry operation. For mixtures where the identification of the masses of individual atoms and molecules that have been ionized are possible to distinguish, mass spectrometry (MS) is a reasonably simple but unique technique 


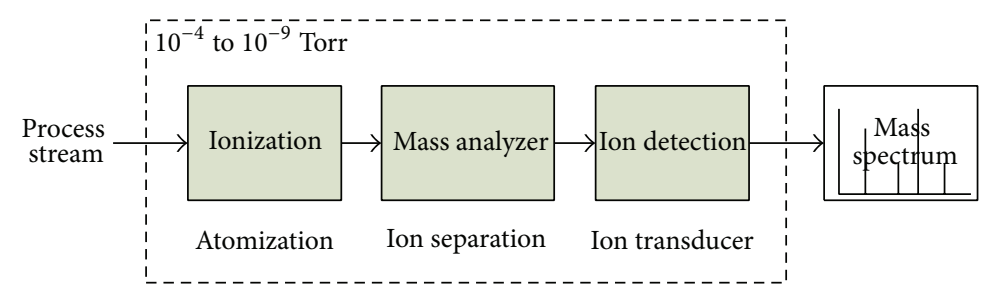

FIGURE 1: Schematic of mass spectroscopy analysis.

in providing fingerprint recognition for the structural and chemical properties of these molecules. A quadrupole mass spectrometer is composed by (i) a sampling system, (ii) a mass spectrometer hardware, and (iii) a data system for acquisition and analysis [7].

The sampling system serves as a connection between the mixture stream under analysis and the vacuum environment that the MS requires. Once the gaseous sample, exiting a column packed with an adsorbent material composing an adsorption separative process, reaches the MS hardware, ionization, separation, and detection take place (Figure 1). During ionization, the sample molecules are turned into ions which are then focused towards the electric quadrupole in order to be detected. The electron impact ionization occurs where a cloud of electrons accelerates towards the ionizer body; the electrons collide with the sample, thus causing the molecular bonds to break and fragment ions to form. Each ion has an identifiable mass. Once the positive ions are formed, they are focused towards the quadrupole mass filter and separated according to their mass-to-charge $(\mathrm{m} / \mathrm{z})$ ratio, where $m$ is the ion mass in atomic mass units (amu or dalton) and $z$ is its charge. The data system displays the ions that pass through the quadrupole and are focused towards a Faraday cup detector. The MS operation depends on maintaining a vacuum pressure $\leq 10^{-4}$ mbar. This pressure should take into account not only the operational pressure of the MS but also the pressure of the sampling system under analysis.

In this paper, an algorithm was developed under an inhouse Labview environment in order to quantify and monitor in real-time the feed or product gaseous streams of continuous separation processes based on columns packed with conventional adsorbent materials such as activated carbon or topical and more recent adsorbents like metal organic frameworks (MOFs). The control system and data acquisition are accomplished, where the adjustment of all parameters that affect sampling, ionization, separation, and detection are controlled. The autotune option is active for a reproducible data acquisition, and peak position and resolution can be optimized according to process conditions. The method approach developed is tuned for the specific mixture and process operating conditions required and can be considered as a real-time standard monitoring/quantification technique for this type of process development applications.

\section{Materials and Methods}

2.1. Materials and MS Conditioning. All gases were provided by Air Liquide (PT): $\mathrm{N}_{2} \mathrm{~N} 45, \mathrm{CO}_{2} \mathrm{~N} 48, \mathrm{CH}_{4} \mathrm{~N} 35$, and $\mathrm{He}$
N50. The composition analysis is performed using a Dymaxion quadrupole mass spectrometer DM100 (Ametek Inc., USA), operated with in-house developed Labview-based software. The standard MS configuration consists of two main components: the open ion source quadrupole mass spectrometer that operates at 1-100 amu mass range and a Faraday cup detector [7]. The unit has a dual-pumping system consisting of a turbomolecular pump and an oil-vane rotary pump (Pfeiffer Vacuum, DE). A manifold heater jacket with temperature controller is also available for degassing of the ionizer chamber. The operating pressure range of the MS system is $10^{-4}$ Torr to ultrahigh vacuum (UHV), with the minimum detectable partial pressure of $5 \times 10^{-12}$ Torr. The MS resolution is adjustable to constant peak width $(0.5 \mathrm{amu}$ at $10 \%$ height). After 30 minutes of warm-up, it shows a mass stability of \pm 0.1 amu and a peak height stability of $\pm 2 \%$. The equipment is linked to the software that provides system integration, autotune capabilities, real-time calculations, and analog/digital I/O RS-232 support. The communication routine is set to 15 milliseconds which allows extremely fast acquisition, command, and response.

Prior to the MS operation, the quadrupole head in the closed MS chamber should be degassed by increasing the filament current in order to burn off any contaminants that may have been collected on the filament. This procedure takes about 10 minutes, after which the MS internal parameters of calibration and ionization of the quadrupole head should be revised and/or tuned and stored.

An automatic calibration adjusts and optimizes the frequency, mass resolution, and mass position, checking also if the current tune parameters are within tolerance. This routine is performed by using residual air to trace two peaks at the low and high ends of the mass range. Usually, for sampling air, the peaks at $m / z 14$ and 40 are followed, though masses 18,28 , and 32 can also be checked. If the internal parameters are out of range, the selected mass peaks are displayed out of position, unresolved and with unsatisfactory sensitivity. Thus, calibration procedure will adjust values of emission current, electron energy, and internal voltages, along with the ranges of peaks resolution and sensitivity. Table 1 presents the range and general user experimental references of the main calibration and ionization parameters, for the MS used in this work.

2.2. MS Analysis for Adsorption Separation Processes. Gas adsorption separation processes, such as Simulated Moving Bed (SMB) or Pressure Swing Adsorption (PSA), as described in Figure 2, are units that operate continuously in a cyclic mode and require the monitoring, quantification, and 
TABLE 1: Main range and user experimental references for the calibration and ionization internal parameters of the MS device employed in this work with mass range of 1-100 amu.

\begin{tabular}{lcc}
\hline Parameter & Standard range [7] & Reference \\
\hline Calibration & & \\
Low position & $-10-10$ & 0.68 \\
High position & $-10-10$ & -0.50 \\
Low resolution & $50-4100$ & 4050 \\
High resolution & $50-4100$ & 1860 \\
Low sensitivity (eV) & $3-10$ & 6 \\
High sensitivity (eV) & $3-10$ & 7 \\
\hline Ionization & & \\
Emission current (mA) & $1-10$ & -70 \\
Electron energy (V) & $-150-30$ & -20 \\
Focus voltage (V) & $-200-10$ & -72 \\
Repeller voltage (V) & $-200-10$ &
\end{tabular}

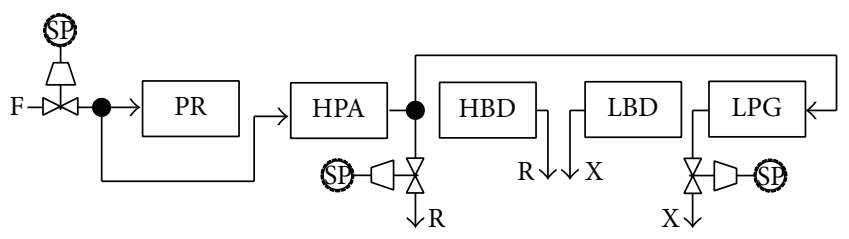

(a) PSA/VSA

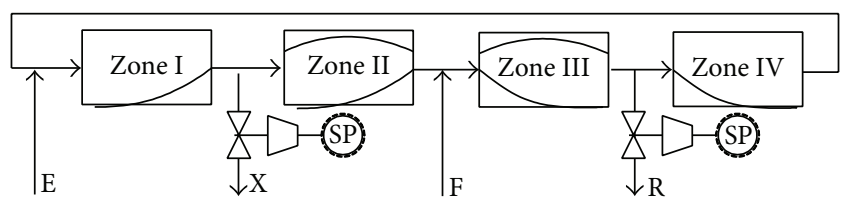

(b) SMB

FIGURE 2: Schematics of adsorption based separation processes, namely, (a) PSA cycle implemented for the $\mathrm{CO}_{2} / \mathrm{N}_{2}$ separation. For the sake of simplicity the scheme only shows the sequential steps of the cycle for one column: Pressurization (PR), High Pressure Adsorption (HPA), High Pressure Blowdown (HBD), Low Pressure Blowdown (LBD), and Low Pressure Purge (LPG). (b) Conventional SMB scheme, which is divided into four zones, one for adding the gaseous desorbent or carrier gas (E), one for withdrawing the slow-migrating adsorbates $(\mathrm{X})$, one for continuous feeding $(\mathrm{F})$, and the last one for withdrawing the fast-migrating adsorbates (R). The positions of the input and output streams are shifted downstream by one column, at fixed intervals, in the direction of gas flow. SP: sampling port.

analysis of their product streams at all times [1-3, 8]. This means that these units have to be coupled to composition analyzers that can respond efficiently in real-time operating conditions.

A regeneration step is followed, where the preferentially adsorbed species are removed as extract (X) product by reducing the total pressure, and the bed is renewed for the next cycle. Although the operation of each bed is batchwise, the whole multibed system is continuously operated in a cyclic steady state (CSS), in which the conditions at the end of each cycle are identical to those at its start. The pressure swing and the CSS movement of the concentration profiles in a mean position inside the bed allow the gas separation to occur.

Several process cycle schemes, steps, and combinations of cycles have been developed [1], based on the system arrangement and the adsorbent material regeneration method. Nitrogen, hydrogen, oxygen, argon, carbon monoxide, carbon dioxide, $n$-paraffins, and methane are candidates for separation/purification by PSA [9-11]. A fully understandable synopsis of PSA patent literature is presented elsewhere $[2,12]$.

The well-established SMB adsorption process was recently reexamined for separation of gases in the light of new developments in novel cyclic operating schemes for liquid-phase operation, with focus on asynchronous mode and cyclic modulation of the internal flow rates [4, 5, 13-15]. Like PSA, SMB is also a continuous adsorptive separation process with numerous applications, many of which are difficult to handle using other separation systems. The conventional SMB (Figure 2(b)) operates at constant pressure and comprises several columns connected in series in a closed- or open-loop configuration. The classical column (synchronous) sequence is divided into four zones of constant flow rates by four ports: one for adding the desorbent or carrier gas (E), one for extracting the slow-migrating adsorbates $(\mathrm{X})$, one for continuous feeding $(\mathrm{F})$, and one for withdrawing the fast-migrating adsorbates (R). The positions of the input and output streams are shifted downstream by one column in order to simulate the movement of a solid bed in the opposite direction to the gas, at fixed time intervals, in the direction of gas flow. Each advancement of the fluid directing device to a new position is called a step, and the completion of all valve steps is called a cycle with a given switching interval of time. Further details on the SMB process operation are reviewed in recent publications $[16,17]$.

The analysis of the gaseous streams in adsorption separation processes is vital, which is reason why the use of the developed method is of extreme importance. The MS sampling system serves as a bridge between the sample environment in PSA or SMB streams and the vacuum environment of $10^{-4}$ to $10^{-9}$ Torr required by the spectrometer.

If a sample is at the same operating pressure as the mass spectrometer, it can be analyzed directly. However, in the application cases reported herein, the sample environment pressures are well above $10^{-4}$ Torr (approx. 4 bar in the SMB and 20-35 bar in the PSA); thus, a pressure reduction sampling system is required through a small-bore capillary that establishes the MS/process continuous linkage. This stream line allows a high-pressurized sample to be delivered directly to the on-line analyzer. Its effective pressure is reduced by using a very small diameter aperture. Note that the sample is flushed through the capillary as a result of a pressure drop in the sample line.

2.3. Method Approach. Mass spectroscopy analysis involves (i) an atomization step with conversion of a substantial fraction of atoms formed to a stream of ions; (ii) the ions separation on the basis of their $m / z$ ratio; and (iii) the measurement of the ion current produced when the ions formed from the sample strike a suitable transducer (Figure 1). Usually, $m / z$ 
is simply the mass of the ion, since most ions in mass spectroscopy are singly charged. Although the operation depends significantly on maintaining low and stable vacuum pressures and on ensuring that operating conditions of the standards and sample are similar, this technique provides fingerprint identification for the structural and chemical properties of the individual atoms and molecules converted to ions.

The mixture-specific algorithm developed provides direct and fully automatic calculation, reporting and monitoring in real-time normalized partial component concentrations of gas streams through mass spectrometry. The primary data is shown in a trend mode, that is, as a pressure-time diagram of the selected masses that are being traced. Binary and ternary gaseous mixtures of $\mathrm{CO}_{2}, \mathrm{CH}_{4}, \mathrm{~N}_{2}$, and He were employed herein as case-studies at both diluted and highly concentrated stream conditions. Although the mixtures chosen are simple in the sense of their spectra analysis, they allow the validation of the method developed for real-time quantification of feed and product stream compositions of continuous gas separation advanced engineering processes. In the studycases where more than one species share the same $m / z$ value of a common ion, one has to consider an amalgam of both of those components on that specific mass.

The gases have different ionization efficiencies in the ionizer of the MS analyzer. Each gas ionizes more or less easily than other gases and the species that ionize more easily show up as higher peaks, that is, with higher partial pressures/ion currents than a gas that is harder to ionize. These gas sensitivities depend on the atomic-molecular reactions occurring in the ionizer due to the electron collisions with the gas molecules. The primary function of the algorithm developed herein is to directly acquire the data necessary to perform the concentration calculations and calculate the sensitivities of the various gases in the mixtures. It is the calculation of the gas sensitivities that drives the real-time algorithm.

Every gas introduced into the MS will exhibit a different affinity to produce ions, that is, an Ionization Probability (IP). When the IPs of two different gases are compared to each other, one can calculate the sensitivity $(S)$ of the gas at the peak corresponding to its mass number by dividing the measured amount of the species (by using any units that are convenient) by its concentration. Towards low gas pressure range of work, the gas sensitivities are generally constant by changing gas pressure; therefore, there is a linear relationship between gas partial pressures and the detected ion currents, $I_{i}$. Consider

$$
I_{i}=\sum_{j=1}^{n_{g}} S_{i j} P_{j},
$$

where $i$ and $j$ are the integer indexes for the ion currents and the gases, respectively, $n_{g}$ is the number of the gases, and $1 \leq i \leq n_{m}$, with $n_{m}$ being the number of the measured ion currents. By doing this simple exercise, one can quickly see that there are huge differences in how the gases produce ions. If one introduces into the MS a known equimolar mixture of $50 / 50 \%$ (-mol) of $\mathrm{CO}_{2} / \mathrm{N}_{2}$ and observes the mass spectrum, the peak corresponding to $\mathrm{CO}_{2}$ would appear to be twice as big as that produced by $\mathrm{N}_{2}$. If one attempts to report the concentrations of these gases based solely on what the mass spectrum looked like, one would calculate that the mixture was $66.7 \% \mathrm{CO}_{2}$ and $33.3 \% \mathrm{~N}_{2}$. Hence, the error in concentrations is due to the differing sensitivities of the gases presented to the MS analyzer. In order to accurately report the concentrations of the mixture, one must correct for the different sensitivities $(S)$ of $\mathrm{N}_{2}$ and $\mathrm{CO}_{2}$. The starting point for this calculation is to assume one of the masses as reference. The species with the lowest molecular weight of the set of components present on the mixture $\left(\mathrm{N}_{2}\right.$ in this example) is usually selected as the reference $(\mathrm{R})$ mass.

Since one was hypothesizing that $\mathrm{CO}_{2}$ is twice as large as $\mathrm{N}_{2}$ based on what the mass spectrum showed us, one would have gathered the following current signal data from the mass spectrum:

$$
I=I_{\mathrm{N}_{2}}+I_{\mathrm{CO}_{2}}, \quad \text { with } I_{\mathrm{CO}_{2}}=2 I_{\mathrm{N}_{2}} \text {. }
$$

Thus, since $I=3 I_{\mathrm{N}_{2}}$, the calculation of the concentrations directly from this information would give

$$
y_{i}(\%)= \begin{cases}\frac{I_{\mathrm{CO}_{2}}}{I}=66.7 & \text { if } i \text { is } \mathrm{CO}_{2} \\ \frac{I_{\mathrm{N}_{2}}}{I}=33.3 & \text { if } i \text { is } \mathrm{N}_{2}\end{cases}
$$

with $y_{i}$ being the average molar fraction of species $i$ (in \%). If the reference species is $\mathrm{N}_{2}$, a correction factor $(f)$ for $\mathrm{CO}_{2}$ must be calculated in order to account for the differing sensitivities $\left(S_{i}\right)$ of the two gases on the equimolar mixture. Consider

$$
\begin{aligned}
& S_{i}= \begin{cases}\frac{I_{\mathrm{CO}_{2}}}{y_{\mathrm{CO}_{2}}}=S_{\mathrm{CO}_{2}} & \text { if } i \text { is } \mathrm{CO}_{2} \\
\frac{I_{\mathrm{N}_{2}}}{y_{\mathrm{N}_{2}}}=\frac{\left(I_{\mathrm{CO}_{2}} / 2\right)}{y_{\mathrm{N}_{2}}}=S_{\mathrm{N}_{2}} & \text { if } i \text { is } \mathrm{N}_{2},\end{cases} \\
& f=\frac{S_{\mathrm{N}_{2}}}{S_{\mathrm{CO}_{2}}}=0.5 .
\end{aligned}
$$

The correction factor for $\mathrm{CO}_{2}$ is then 0.5 and should be applied to any subsequent reading taken for $\mathrm{CO}_{2}$. Therefore, the correct MS signal $\left(I^{\prime}\right)$ is

$$
I_{i}^{\prime}= \begin{cases}I_{\mathrm{CO}_{2}} f & \text { if } i \text { is } \mathrm{CO}_{2} \\ I_{\mathrm{N}_{2}} & \text { if } i \text { is } \mathrm{N}_{2} .\end{cases}
$$

Note that there is no correction factor applied to $\mathrm{N}_{2}$, since this component was chosen as the reference. Given the correct ion current,

$$
I^{\prime}=I_{\mathrm{N}_{2}}^{\prime}+I_{\mathrm{CO}_{2}}^{\prime}=I_{\mathrm{N}_{2}}(1+2 f) \text {. }
$$

The correct concentrations would then be

$$
y_{i}^{\prime}(\%)= \begin{cases}\frac{I_{\mathrm{CO}_{2}}^{\prime}}{I^{\prime}}=\frac{2 f}{(1+2 f)}=50 & \text { if } i \text { is } \mathrm{CO}_{2} \\ \frac{I_{\mathrm{N}_{2}}^{\prime}}{I^{\prime}}=\frac{1}{(1+2 f)}=50 & \text { if } i \text { is } \mathrm{N}_{2} .\end{cases}
$$




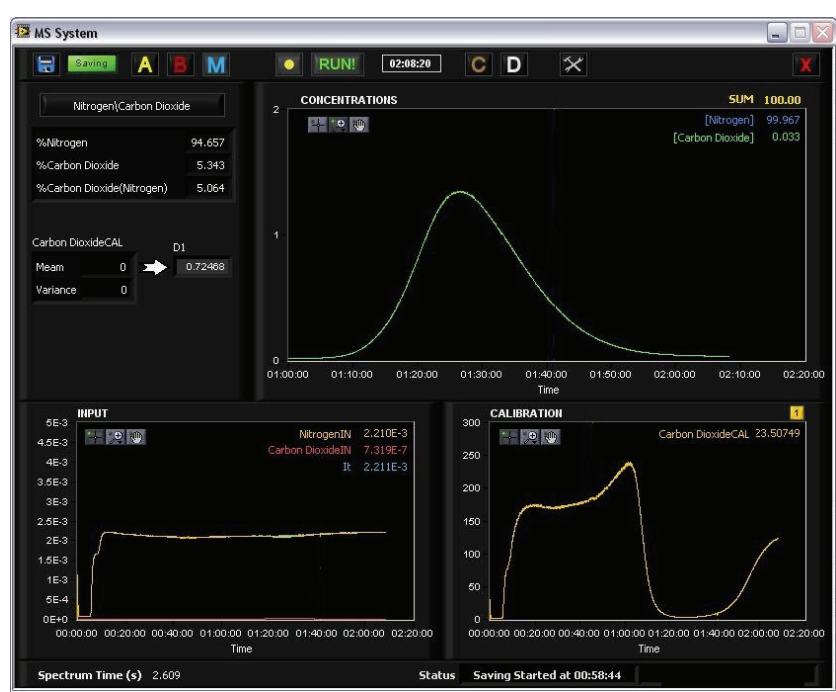

Figure 3: Illustration of the graphical interface of the algorithm developed.

Now it is possible to vary the concentrations of these gases from approximately $0.1 \%$ to $100 \%(-\mathrm{mol})$ and be able to report the concentration of the mixture accurately.

The algorithm developed accounts at real-time for the correction calculations described above and is presented as a single file that produces three direct graphical screens to the user. Figure 3 illustrates the graphical interface built comprising the algorithm developed. The first data screen (INPUT graph) carries a number of operators which reports (i) the mass signals that track the ions of interest, (ii) the components sensitivities referenced to species $\mathrm{R}$, that is, the correction factors applied to reference R, and (iii) the total corrected ion signals for all measured components of the mixture in study.

The second screen (CALIBRATION graph) allows the user to enter the species sensitivity values obtained in screen 1. Once this occurs, the data are automatically corrected in the calculation sequence. A third screen (CONCENTRATIONS graph) reports the calculated concentrations following (8). There are no user entries necessary to view the gaseous concentration profiles entering or exiting the adsorption process at real-time in this final screen. The algorithm is saved after a refined single-point calibration, in a file which depends on the gases composing the mixture and their concentration range.

\section{Results and Discussion}

3.1. Single-Point Calibration. The pure component mass spectra were primarily performed in order to obtain the constant relation of its composing ion masses, that is, the constant relative intensities of the fragment ions composing each species (also called cracking pattern). The experiments on pure $\mathrm{CO}_{2}$, $\mathrm{CH}_{4}, \mathrm{~N}_{2}$, and He were repeated at least three times in order to check reproducibility and compared with references from the NIST Mass Spectrometry Data Center [18].

The ionized $\mathrm{CO}_{2}$ molecule appears at $m / z=44$, though a fraction of the ions appear in the spectrum at $m / z$ values

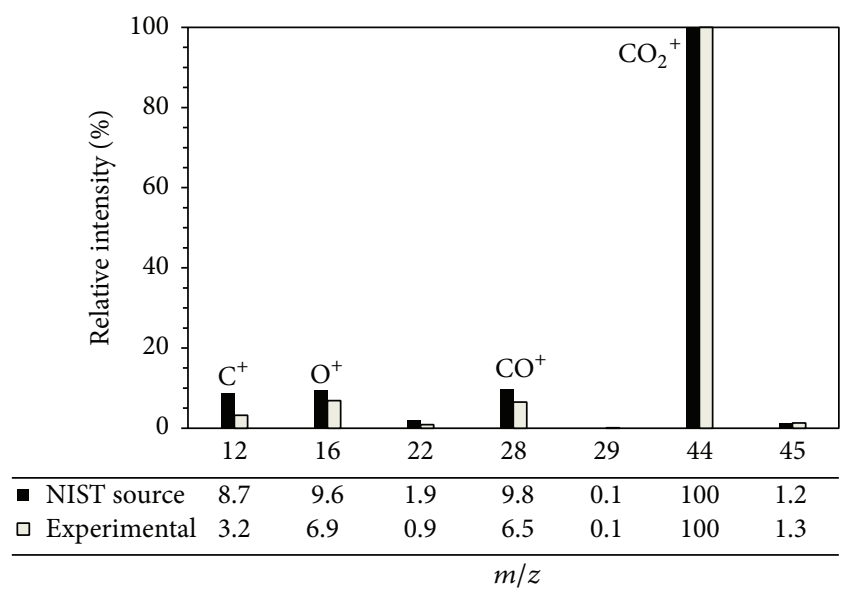

FIgURE 4: Pure $\mathrm{CO}_{2}$ mass spectrum obtained experimentally and its relative ion intensities. Reference values from NIST Chemistry WebBook [18] are shown for comparing.

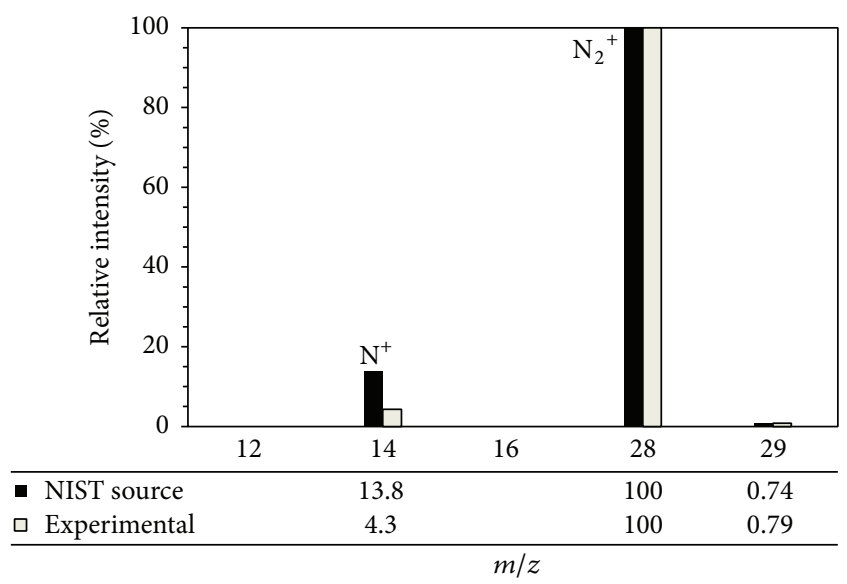

FIgURe 5: Pure $\mathrm{N}_{2}$ mass spectrum obtained experimentally and its relative ion intensities. Reference values from NIST Chemistry WebBook [18] are shown for comparing.

lower than 44. Thus, a constant relative ion intensities (in \%) for the pure $\mathrm{CO}_{2}$ species was obtained experimentally and used as a reference state (Figure 4). Cleavage of a C-O bond in the molecule to produce ionized carbon monoxide $\left(\mathrm{CO}^{+}\right)$ or ionized atomic oxygen $\left(\mathrm{O}^{+}\right)$results in the fragment ions at $m / z$ s of 28 and 16, respectively. Likewise, the loss of two neutral oxygen atoms results in an additional fragment at $m / z=12$ for carbon $\left(\mathrm{C}^{+}\right)$. The molecular ion is designated as $\mathrm{CO}_{2}{ }^{+}$and the fragment ions are named as $\mathrm{CO}^{+}, \mathrm{O}^{+}$, and $\mathrm{C}^{+}$. In order to standardize mass spectra for pure compounds, the total ion intensity relative to the base peak normalized to 100 (i.e., $\mathrm{m} / z=44$ for $\mathrm{CO}_{2}$ ) is employed. From the total relative ion intensity observed experimentally for $\mathrm{CO}_{2}$, averages of $1.9,4.7,0.8,5.0,0.1,78.5$, and $1 \%$ corresponded to mass numbers $m / z$ of $12,16,22,28,29,44$, and 45 , respectively.

The nitrogen spectrum obtained experimentally shows mainly two typical peaks for ion masses, $\mathrm{m} / z, 14$ and 28 , relative to fragment ion $\mathrm{N}^{+}$and molecular ion $\mathrm{N}_{2}^{+}$, respectively. Figure 5 presents the pure $\mathrm{N}_{2}$ mass spectrum 


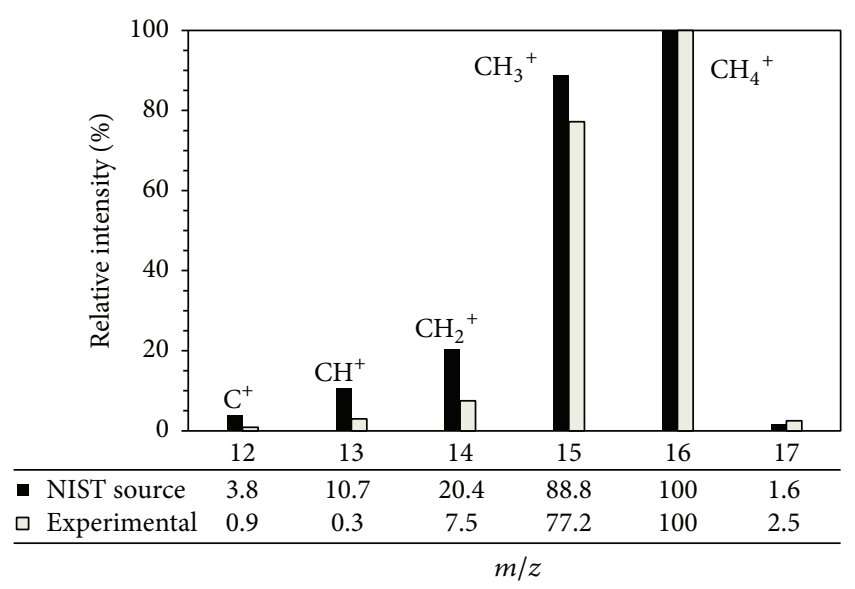

FIgURE 6: Pure $\mathrm{CH}_{4}$ mass spectrum obtained experimentally and its relative ion intensities. Reference values from NIST Chemistry WebBook [18] are shown for comparing.

obtained experimentally and their relative ion intensities. As a standard reference, from the total ion masses observed experimentally, $3.9 \%$ corresponds to $\mathrm{m} / z=14$ and $90.9 \%$ to $m / z=28$, against the reference numbers of $4.2 \%$ and $93.3 \%$, respectively.

Methane experimental spectrum shows total ion average intensities, relative to the base peak (at $m / z=16$ ), of 0.6 , $1.8,8.8,38.0,46.8$, and $1.3 \%$ for the mass numbers $m / z$ of $12,13,14,15,16$, and 17, respectively. Figure 6 shows the pure $\mathrm{CH}_{4}$ mass spectra obtained experimentally and their relative ion intensities. The concordant reference total intensities values for the respective ion masses are 1.0, 4.1, 8.2, 44.0, 51.2, and $0.5 \%$, respectively. The differences observed are probably due to distinct impurities on the gas source and the accuracy of the MS analyzers for both the reference and experimental measurements. Helium is monoatomic and has a high ionization potential. Therefore, the observed helium ion intensities are quite small and its spectrum shows typically a base peak normalized to $100 \%$ at mass $m / z=4$.

A typical calibration sequence is composed by the algorithm described above (2)-(8). Note that if one is going to use the MS to monitor the effluent from a separation process, the software routine should be previously launched and let running for at least one hour in order to scan the masses of interest just as one have them in a regular process stream before calibration attempt. This will allow the system to come to a mechanical, electrical, and thermal equilibrium that is essential to system stability. In the cases where more than one species of the gas mixtures employed in the single-point calibrations share the same ion masses, the species contribution to those common masses should be distinguished in order to properly quantify the streams and employ the right correction factor $f$. For example, when a $\mathrm{CO}_{2} / \mathrm{N}_{2}$ mixture is considered, the traceable mass numbers $m / z$ to identify the peaks of the different gases are 44 and 28, respectively. However, the spectral overlap of mass 28 has to be resolved prior to the stream quantification. From the total relative ion intensity observed experimentally for $\mathrm{CO}_{2}$, the averaged percentage of mass 28 relative to mass 44 is $6.5 \%$ (Figure 4 ), the rest being relative to the presence of $\mathrm{N}_{2}$ in the mixture. Therefore, the corrected $\mathrm{N}_{2}$ concentration should discount the ion mass 28 relative to the pure $\mathrm{CO}_{2}$ spectra, and the $\mathrm{CO}_{2}$ concentration must be determined through a correction factor calculated on the same basis:

$$
\begin{aligned}
& y_{i}^{\prime}(\%)= \begin{cases}\frac{I_{\mathrm{CO}_{2}}^{\prime}}{I^{\prime}}=\frac{m_{44}^{\mathrm{CO}_{2}} f}{I^{\prime}} & \text { if } i \text { is } \mathrm{CO}_{2} \\
\frac{I_{\mathrm{N}_{2}}^{\prime}}{I^{\prime}}=\frac{\left(m_{28}-m_{28}^{\mathrm{CO}_{2}}\right)}{I^{\prime}} & \text { if } i \text { is } \mathrm{N}_{2}\end{cases} \\
& I^{\prime}=I_{\mathrm{N}_{2}}^{\prime}+I_{\mathrm{CO}_{2}}^{\prime}=\left(m_{28}-m_{28}^{\mathrm{CO}_{2}}\right)+m_{44}^{\mathrm{CO}_{2}} f,
\end{aligned}
$$

with $f=\left(m_{28}-m_{28}^{\mathrm{CO}_{2}}\right) / m_{44}^{\mathrm{CO}_{2}} y_{\mathrm{CO}_{2}} / y_{\mathrm{N}_{2}}$ and $m_{28}=m_{28}^{\mathrm{N}_{2}}+$ $m_{28}^{\mathrm{CO}_{2}}=m_{28}^{\mathrm{N}_{2}}+0.065 \cdot m_{44}^{\mathrm{CO}_{2}}$, where $m_{i}^{j}$ represents the intensity current of the ion mass number $i$ relative to its gas molecule $j$, $m_{i}$ denotes the total intensity current of the ion mass number $i$, and $y_{\mathrm{CO}_{2}} / y_{\mathrm{N}_{2}}$ is the molar fraction ratio of the mixture composition at the single-point calibration. A more complex example is the ternary mixture $\mathrm{CO}_{2} / \mathrm{CH}_{4} / \mathrm{N}_{2}$, where there is spectral overlap of both masses 16 and 28 that have to be resolved prior to stream quantification. The species corrected concentrations would be

$$
\begin{aligned}
& y_{i}^{\prime}(\%)= \begin{cases}\frac{I_{\mathrm{CO}_{2}}^{\prime}}{I^{\prime}}=\frac{m_{44}^{\mathrm{CO}_{2}} f_{1}}{I^{\prime}} & \text { if } i \text { is } \mathrm{CO}_{2} \\
\frac{I_{\mathrm{N}_{2}}^{\prime}=\frac{\left(m_{28}-m_{28}^{\mathrm{CO}_{2}}\right) f_{2}}{I^{\prime}}}{I^{\prime}} & \text { if } i \text { is } \mathrm{N}_{2} \\
\frac{I_{\mathrm{CH}_{4}}^{\prime}=\frac{\left(m_{16}-m_{16}^{\mathrm{CO}_{2}}\right)}{I^{\prime}}}{I^{\prime}} & \text { if } i \text { is } \mathrm{CH}_{4}\end{cases} \\
& I^{\prime}=I_{\mathrm{CO}_{2}}^{\prime}+I_{\mathrm{N}_{2}}^{\prime}+I_{\mathrm{CH}_{4}}^{\prime} \\
& =m_{44}^{\mathrm{CO}_{2}} f+\left(m_{28}-m_{28}^{\mathrm{CO}_{2}}\right) f_{2} \\
& +\left(m_{16}-m_{16}^{\mathrm{CO}_{2}}\right) \text {, }
\end{aligned}
$$

with $f_{1}=\left(m_{16}-m_{16}^{\mathrm{CO}_{2}}\right) / m_{44}^{\mathrm{CO}_{2}} y_{\mathrm{CO}_{2}} / y_{\mathrm{CH}_{4}}$ and $f_{2}=\left(m_{16}-\right.$ $\left.m_{16}^{\mathrm{CO}_{2}}\right) /\left(m_{28}-m_{28}^{\mathrm{CO}_{2}}\right) y_{\mathrm{N}_{2}} / y_{\mathrm{CH}_{4}}$, the correction factors for $\mathrm{CO}_{2}$ and $\mathrm{N}_{2}$, respectively. The reference species is $\mathrm{CH}_{4}$ and $m_{16}=$ $m_{16}^{\mathrm{CH}_{4}}+m_{16}^{\mathrm{CO}_{2}}=m_{16}^{\mathrm{CH}_{4}}+0.069 \cdot m_{44}^{\mathrm{CO}_{2}}$, considering that the averaged percentage of mass 16 relative to mass 44 is $6.9 \%$ (Figure 4). Once again, $y_{\mathrm{CO}_{2}} / y_{\mathrm{CH}_{4}}$ and $y_{\mathrm{N}_{2}} / y_{\mathrm{CH}_{4}}$ are the molar fraction ratios of the mixture composition employed at the single-point calibration.

After the determination of the proper correction factor $f$, a mixture composition distinct from the one employed initially in the single-point calibration, is then fed to the MS in order to check the correctness of the protocol. These gas mixtures are set by high precision mass-flow controllers (error $< \pm 1 \%$ of full scale range), one for each species composing the mixture of interest, and capable of producing accurate gas stream compositions. All the procedure is then repeated and $f$ value is refined until the final species concentrations, calculated from the algorithm, match the feed stream entering the MS. Usually, it is assumed that 
TABLE 2: Illustrative data results obtained for the single-point calibration procedure of the sampling gas mixtures used experimentally. Run 1 reports the single point calibration experiment, while runs 2 and 3 denote the respective tuning runs.

\begin{tabular}{|c|c|c|c|c|c|c|c|}
\hline \multirow{2}{*}{ Mixture } & \multicolumn{3}{|c|}{ Run 1} & \multicolumn{2}{|c|}{ Run 2} & \multicolumn{2}{|c|}{ Run 3} \\
\hline & $y_{\mathrm{in}_{i}}$ & $f_{i}$ & $y_{\mathrm{ms}_{i}}$ & $y_{\mathrm{in}_{i}}$ & $y_{\mathrm{ms}_{i}}$ & $y_{\mathrm{in}_{i}}$ & $y_{\mathrm{ms}_{i}}$ \\
\hline $\mathrm{CH}_{4}$ & 1.05 & 0.752 & $1.05(-)$ & 0.44 & $0.44(-)$ & 0.22 & $0.23(0.01)$ \\
\hline $\mathrm{N}_{2}{ }^{*}$ & 98.94 & - & $98.95(0.01)$ & 99.56 & $99.57(0.01)$ & 99.78 & $99.77(-0.01)$ \\
\hline $\mathrm{CH}_{4}$ & 1.00 & 0.205 & $1.00(-)$ & 0.38 & $0.39(0.01)$ & 4.02 & $4.17(0.15)$ \\
\hline $\mathrm{He}^{*}$ & 99.00 & - & $99.00(-)$ & 99.62 & $99.61(-0.01)$ & 95.98 & $95.83(-0.15)$ \\
\hline $\mathrm{CH}_{4}$ & 19.91 & 0.208 & $19.99(0.08)$ & 69.33 & $70.98(1.65)$ & - & - \\
\hline $\mathrm{He}^{*}$ & 80.09 & - & $80.01(-0.08)$ & 30.67 & $29.02(-1.65)$ & - & - \\
\hline $\mathrm{CO}_{2}$ & 8.91 & 0.862 & $8.92(0.01)$ & 0.91 & $1.05(0.14)$ & 14.26 & $15.26(1.00)$ \\
\hline $\mathrm{N}_{2}{ }^{*}$ & 91.07 & - & $91.08(0.01)$ & 99.09 & $98.95(-0.14)$ & 85.75 & $84.74(1.01)$ \\
\hline $\mathrm{CO}_{2}$ & 20.01 & 0.536 & $19.97(-0.04)$ & 49.91 & $47.84(-2.07)$ & 10.04 & $8.63(-1.41)$ \\
\hline $\mathrm{N}_{2}{ }^{*}$ & 79.99 & - & $80.03(0.04)$ & 50.09 & $52.16(2.07)$ & 89.96 & $91.36(1.40)$ \\
\hline $\mathrm{CO}_{2}$ & 9.02 & 0.126 & $9.07(0.05)$ & 4.76 & $4.48(-0.28)$ & - & - \\
\hline $\mathrm{He}^{*}$ & 90.98 & - & $90.92(-0.06)$ & 95.24 & $95.52(0.28)$ & - & - \\
\hline $\mathrm{CO}_{2}$ & 50.13 & 0.106 & $50.12(-0.01)$ & 75.22 & $75.13(-0.09)$ & - & - \\
\hline $\mathrm{He}^{*}$ & 49.87 & - & $49.88(0.01)$ & 24.78 & $24.87(0.09)$ & - & - \\
\hline$\overline{\mathrm{N}_{2}}$ & 29.89 & 0.126 & $30.03(0.14)$ & 79.78 & $77.15(-2.63)$ & - & - \\
\hline $\mathrm{He}^{*}$ & 70.13 & - & $69.97(-0.16)$ & 20.22 & $22.85(2.63)$ & - & - \\
\hline $\mathrm{N}_{2}$ & 49.94 & 0.128 & $50.20(0.26)$ & 39.49 & $39.23(-0.26)$ & - & - \\
\hline $\mathrm{He}^{*}$ & 50.06 & - & $49.98(-0.08)$ & 60.51 & $60.77(0.26)$ & - & - \\
\hline $\mathrm{N}_{2}{ }^{*}$ & 55.35 & - & $55.27(-0.08)$ & 72.66 & $75.56(2.90)$ & 55.32 & $55.77(0.45)$ \\
\hline $\mathrm{CH}_{4}$ & 8.68 & 0.762 & $8.71(0.03)$ & 11.42 & $8.63(-2.79)$ & 8.69 & $8.73(0.04)$ \\
\hline $\mathrm{CO}_{2}$ & 35.96 & 0.628 & $36.02(-0.06)$ & 15.92 & $15.81(0.11)$ & 35.99 & $35.50(-0.49)$ \\
\hline
\end{tabular}

${ }^{*}$ Reference (R): species of the gas mixture.

Values in brackets denote the offset (in \%) between inlet/outlet MS streams at $309 \mathrm{~K}$.

differences of ca. $\pm 0.01-0.25 \%$, between the generated (by the mass-flow controllers) and detected (by the MS) stream mole fraction compositions, are sufficient to accept the calibration as valid. The variance relative to this range depends on the partial compositions of the calibration mixture relative to the ones chosen for tuning, that is, the calibration error increases as both mixture compositions are more distinct. Table 2 illustrates data results for the single-point calibration procedure of the sampling gas mixtures used experimentally. Run 1 reports the calibration experiment, while 2 and 3 denote the subsequent tuning runs. Note that $y_{\mathrm{in}_{i}}$ is the average molar fraction of species $i$ (in \%) fed to the MS by the mass-flow controllers, and $y_{\mathrm{ms}_{i}}$ is the average molar fraction of species $i$ (in \%) quantified by the analyzer, by employing the algorithm developed. The data obtained show that the algorithm employed in the methodology described is validated by the good agreement between the known gas compositions fed to the MS and their quantification by the spectrometer. Also, it is shown that, whenever possible, the MS calibration procedure should be performed with mixture compositions as similar as (or within a close range) the ones of the streams to be quantified in process applications. Correctly, a refined calibration should be performed for the gaseous system under study, before each gas-phase inlet/exit stream quantification, in any separation process. This ensures the best approximation to the real gas stream compositions during regular process operation.
3.2. Real-Time Composition Analysis of Process Gas Streams. The validation of the algorithm here described was also performed under the development of continuous PSA and SMB gas separation process schemes, in order to quantify and monitor at real-time the feed or product gaseous streams of interest. The method developed was tuned for the separation of gas mixtures such as $\mathrm{CO}_{2} / \mathrm{N}_{2}$ and $\mathrm{CO}_{2} / \mathrm{CH}_{4} / \mathrm{N}_{2}$.

Figure 7 presents the experimental and simulated temporal profiles of total molar flow rate $(Q)$, inlet composition $\left(y_{i_{\text {in }}}\right)$, and outlet composition $\left(y_{i_{\text {out }}}\right)$ of the gas flowing through a single SMB column setup for the three cycles of operation of a classical 4-column SMB (synchronous) operating mode [5]. The experimental data was obtained at realtime through the MS and following the methodology developed. The simulation results were obtained using nonlinear programming formulated in AMPL [19] and solved using an efficient interior-point solver [20-23]. The optimized SMB system is reported for the separation of $\mathrm{CH}_{4} / \mathrm{CO}_{2}$, carried out experimentally at $309 \mathrm{~K}$ of temperature and 4 bar of total pressure, which remains constant in time over each switching interval $(\tau)$ of the cycle. The feed stream comprises a diluted mixture of $0.5 \%(-\mathrm{mol}) \mathrm{CO}_{2}$ and $0.5 \%(-\mathrm{mol}) \mathrm{CH}_{4}$ in $\mathrm{N}_{2}$, in order to place the separation in the linear adsorption range. Nitrogen is the mobile phase, acting as diluting carrier gas for the feed stream and as eluent. Based on the selectivity for the linear range of this separation problem $\left(K_{\mathrm{CO}_{2}} / K_{\mathrm{CH}_{4}}=2.9\right)$, its difficulty can be loosely classified in the easy-to-moderate 

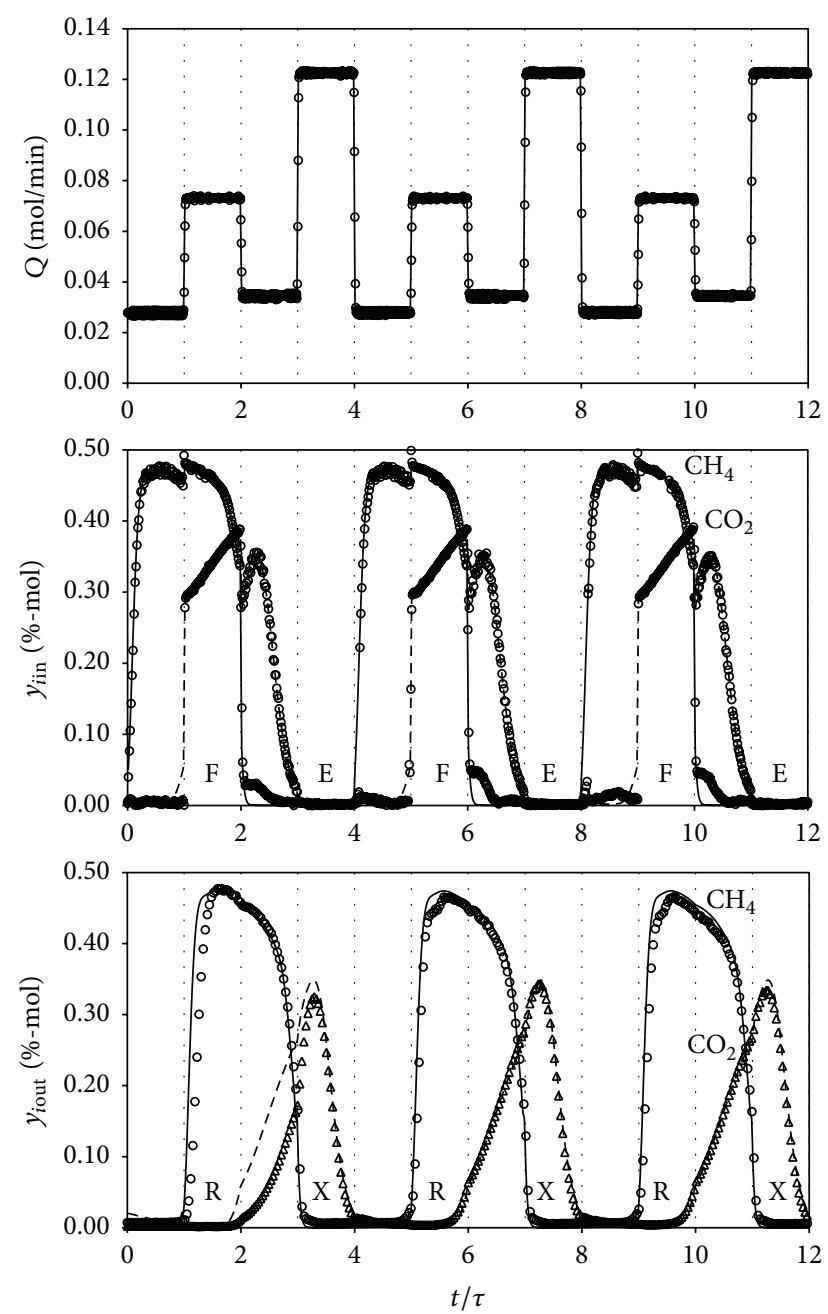

FIgURE 7: Experimental (symbols) and simulated (lines) temporal profiles of total molar flow rate $(Q)$, inlet composition $\left(y_{i \text { in }}\right)$, and outlet composition $\left(y_{\text {iout }}\right)$ of the gas flowing through a column for the 3 cycles of a classical 4-column SMB (synchronous) operation: $\mathrm{CH}_{4} / \mathrm{CO}_{2}$ in $\mathrm{N}_{2}, 309 \mathrm{~K}$, and 4 bar. Every cycle, fresh feed, and eluent are added during $1<t / \tau<2$ and $3<t / \tau<4$, respectively; extract is obtained at $1<t / \tau<2$ and raffinate is withdrawn at $3<t / \tau<4$. The optimized feed flow rate is $Q_{\mathrm{F}}=38.9 \mathrm{mmol} / \mathrm{min}$ for a specific eluent consumption of $Q_{E} / Q_{F}=2.44$ and a switching interval of the cycle of $\tau=9.22[5]$.

range [1]. Nevertheless, the choice is suitable for demonstrating the feasibility of the process [5] and validating the experimental stream quantification by the sensitive mass spectroscopy methodology developed. The composition of the outlet gaseous effluents determined through the MS quantification method reproduces completely the simulated profiles, which provides further evidence that our model and setup capture the main features of the separation process and that it can be confidently employed in advanced separation processes $[5,6]$.

The sensitive MS methodology was also tested in a typical experimental PSA cycle of ca. 14 min which was implemented for the separation of a feed stream composed by $30 \%$ (-mol) $\mathrm{CO}_{2}$ and $70 \%(-\mathrm{mol}) \mathrm{N}_{2}$ mixture at $309 \mathrm{~K}$ of temperature [23].
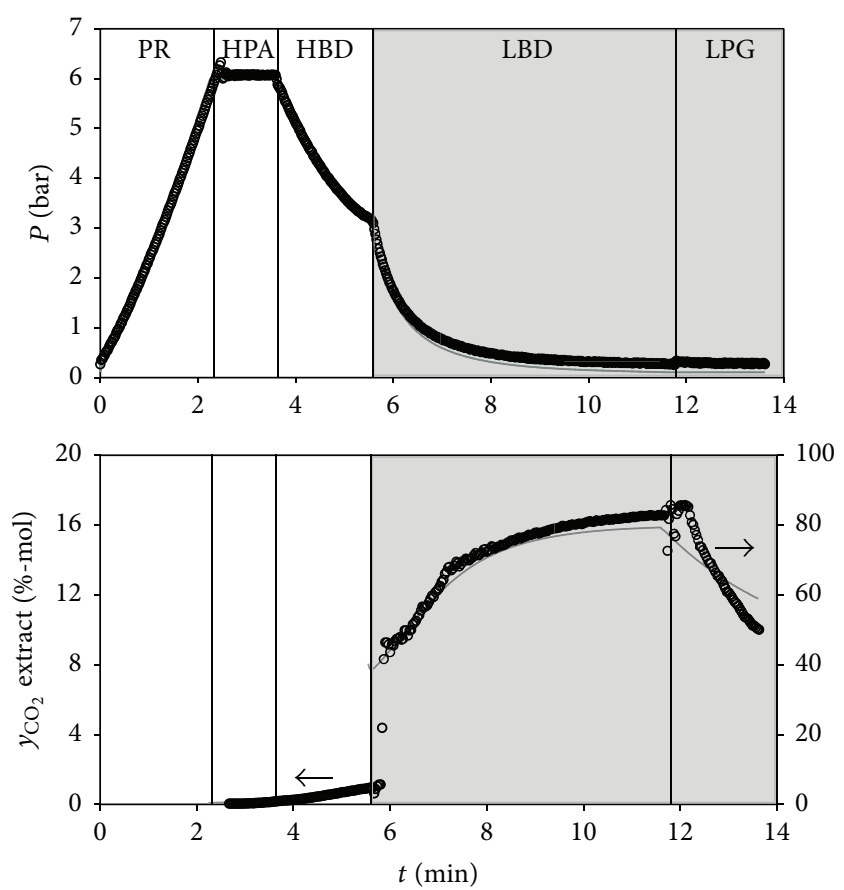

FIGURE 8: Comparison between the predicted (lines) and the experimental (symbols) pressure and $\mathrm{CO}_{2}$ effluent composition histories for a PSA run at CSS employed for the separation of $\mathrm{CO}_{2} /$ $\mathrm{N}_{2} 30: 70 \%$ (-mol) mixture at $309 \mathrm{~K}, P_{\mathrm{H}}=6$ bar, $P_{\mathrm{L}}=0.4 \mathrm{bar}$, feed flow rate of $3.0 \mathrm{slpm}$, and a total feed/cycle of $10.75 \mathrm{~L}$ STP (STP: $273 \mathrm{~K}, 1 \mathrm{~atm})$ [23]. White area denotes cocurrent operation with raffinate $\left(\mathrm{N}_{2}\right)$ production, whereas grey area reflects the countercurrent operation with extract $\left(\mathrm{CO}_{2}\right)$ collecting.

Figure 8 illustrates the comparison between the predicted (lines) and the experimental (symbols) pressure and $\mathrm{CO}_{2}$ effluent composition histories for a PSA run at CSS. The periodic cycle comprises five steps: (1) Pressurization (PR) up to $P_{\mathrm{H}}=6$ bar of pressure; (2) adsorption (HPA) at $P_{\mathrm{H}}$; (3) blowdown (HBD) down to $P_{m}=3.2$ bar; (4) blowdown (LBD) down to $P_{\mathrm{L}}=0.3 \mathrm{bar}$; (5) and purge (LPG) at $P_{\mathrm{L}}$. During $\mathrm{HPA}$ and $\mathrm{HBD}, \mathrm{N}_{2}$ product is obtained, while during LBD and LPG, $\mathrm{CO}_{2}$ is produced. Modelling details are shown elsewhere [23]. Results report a very good agreement between both simulation and experimental profiles, which proves the feasibility of the method developed for the quantification of experimental stream compositions.

\section{Conclusions}

In this work, we have reported a new sensitive and specific method approach by using an algorithm developed for the assay of gas composition monitoring and quantification analysis in both inlet/feed and outlet/product streams of continuous advanced separation processes. The experimental feasibility and effectiveness of this procedure were assessed by running and comparing optimized process configurations for the separation of gas mixtures of $\mathrm{CO}_{2}, \mathrm{CH}_{4}$, and $\mathrm{N}_{2}$ based on columns packed with conventional adsorbent materials such as activated carbon or topical and more recent adsorbents like 
metal organic frameworks (MOFs). To our knowledge, it is the only methodology reported in detail for these applications providing full description and validation data, with accuracy and precision within $0.01-0.25 \%(-\mathrm{mol})$ for 0.1 to $100 \%$ (mol) composition mixtures. The algorithm here developed can be applied as a standard and routine chromatographic analysis technique. Its successful application to gas mixtures of $\mathrm{CO}_{2}, \mathrm{CH}_{4}$, and $\mathrm{N}_{2}$ demonstrated that it is suitable for the research and development, analysis, and monitoring of feed and product streams of continuous gas separation processes, such as competitive PSA and SMB.

\section{Competing Interests}

The authors declare that there are no competing interests regarding the publication of this paper and regarding the funding that they have received.

\section{Acknowledgments}

The authors acknowledge FCT/MEC for funding through projects PTDC/AAC-AMB/108849/2008, EXCL/QEQ-PRS/ 0308/2012, UID/QUI/50006/2013, and ELAC2014/BEE0367. R. P. P. L. Ribeiro and I. A. A. C. Esteves acknowledge FCT/ MCTES for financial support through Grant SFRH/BPD/ 103533/2014 and FCT Investigator Contract IF/01016/2014, respectively. This work was partially supported by the Associate Laboratory Research Unit for Green Chemistry, Technologies and Processes Clean, LAQV which is financed by national funds from FCT/MCTES (UID/QUI/50006/2013) and cofinanced by the ERDF under the PT2020 Partnership Agreement (POCI-01-0145-FEDER-007265).

\section{References}

[1] R. T. Yang, Gas Separation by Adsorption Processes, Butterworths, 1987.

[2] D. M. Ruthven, Principles of Adsorption and Adsorption Processes, John Wiley \& Sons, 1984.

[3] S. Farooq, D. M. Ruthven, and K. S. Kneabel, Pressure Swing Adsorption, VCH Publishers, 1994.

[4] I. A. A. C. Esteves and J. P. B. Mota, "Gas separation by a novel hybrid membrane/pressure swing adsorption process," Industrial \& Engineering Chemistry Research, vol. 46, no. 17, pp. 57235733, 2007.

[5] J. P. B. Mota, I. A. A. C. Esteves, and M. F. J. Eusébio, "Synchronous and asynchronous SMB processes for gas separation," AIChE Journal, vol. 53, no. 5, pp. 1192-1203, 2007.

[6] J. P. B. Mota and I. A. A. C. Esteves, "Optimal design and experimental assessment of time-variable simulated moving bed for gas separation," Industrial \& Engineering Chemistry Research, vol. 46, no. 21, pp. 6978-6988, 2007.

[7] Dymaxion Quadrupole Mass Spectrometer Manual, Ametek Process Instruments, Pittsburgh, Pa, USA, 2004.

[8] G. Guiochon, A. Felinger, A. M. Katti, and D. G. Shirazi, Fundamentals of Preparative and Nonlinear Chromatography, Elsevier, Amsterdam, The Netherlands, 2nd edition, 2006.

[9] D. Basmadjian and L. A. Pogorski, US Patent 3,279,153, 1966.
[10] S. Sircar, "Separation of multicomponent gas mixtures," US Patent 4,171,206, 1979.

[11] R. Kumar, S. Sircar, T. R. White, and E. J. Greskovich, US Patent 4,477,265, 1984.

[12] D. Tondeur and P. C. Wankat, "Gas purification by pressure swing adsorption," Separation \& Purification Methods, vol. 14, no. 2, pp. 157-212, 1985.

[13] K. P. Kostroski and P. C. Wankat, "Separation of dilute binary gases by simulated-moving bed with pressure-swing assist: SMB/PSA processes," Industrial \& Engineering Chemistry Research, vol. 47, no. 9, pp. 3138-3149, 2008.

[14] K. P. Kostroski and P. C. Wankat, "Separation of concentrated binary gases by hybrid pressure-swing adsorption/simulatedmoving bed processes," Industrial \& Engineering Chemistry Research, vol. 48, no. 9, pp. 4445-4465, 2009.

[15] M. C. Campo, M. C. Baptista, A. M. Ribeiro et al., "Gas phase SMB for propane/propylene separation using enhanced $13 \mathrm{X}$ zeolite beads," Adsorption, vol. 20, no. 1, pp. 61-75, 2014.

[16] C. Y. Chin and N.-H. L. Wang, "Simulated moving bed equipment designs," Separation \& Purification Reviews, vol. 33, no. 2, pp. 77-155, 2004.

[17] P. S. Gomes and A. E. Rodrigues, "Simulated moving bed chromatography: from concept to proof-of-concept," Chemical Engineering \& Technology, vol. 35, no. 1, pp. 17-34, 2012.

[18] U.S. Secretary of Commerce, Standard Reference Database Number 69: The NIST Chemistry WebBook, December 2015, http://webbook.nist.gov/chemistry/.

[19] R. Fourer, D. M. Gay, and B. W. Kernighan, AMPL: A Modeling Language for Mathematical Programming, Brooks/ColeThomson Learning, Pacific Grove, Calif, USA, 2nd edition, 2003.

[20] A. Wächter and L. T. Biegler, "On the implementation of an interior-point filter line-search algorithm for large-scale nonlinear programming," Mathematical Programming, vol. 106, no. 1, pp. 25-57, 2006.

[21] Y. Kawajiri and L. T. Biegler, "Optimization strategies for simulated moving bed and powerfeed processes," AIChE Journal, vol. 52, no. 4, pp. 1343-1350, 2006.

[22] Y. Kawajiri and L. T. Biegler, "Nonlinear programming superstructure for optimal dynamic operations of simulated moving bed processes," Industrial and Engineering Chemistry Research, vol. 45, no. 25, pp. 8503-8513, 2006.

[23] I. A. A. C. Esteves and J. P. B. Mota, "Hybrid membrane/PSA processes for $\mathrm{CO}_{2} / \mathrm{N}_{2}$ separation," Adsorption Science \& Technology, vol. 25, no. 9, pp. 693-715, 2007. 

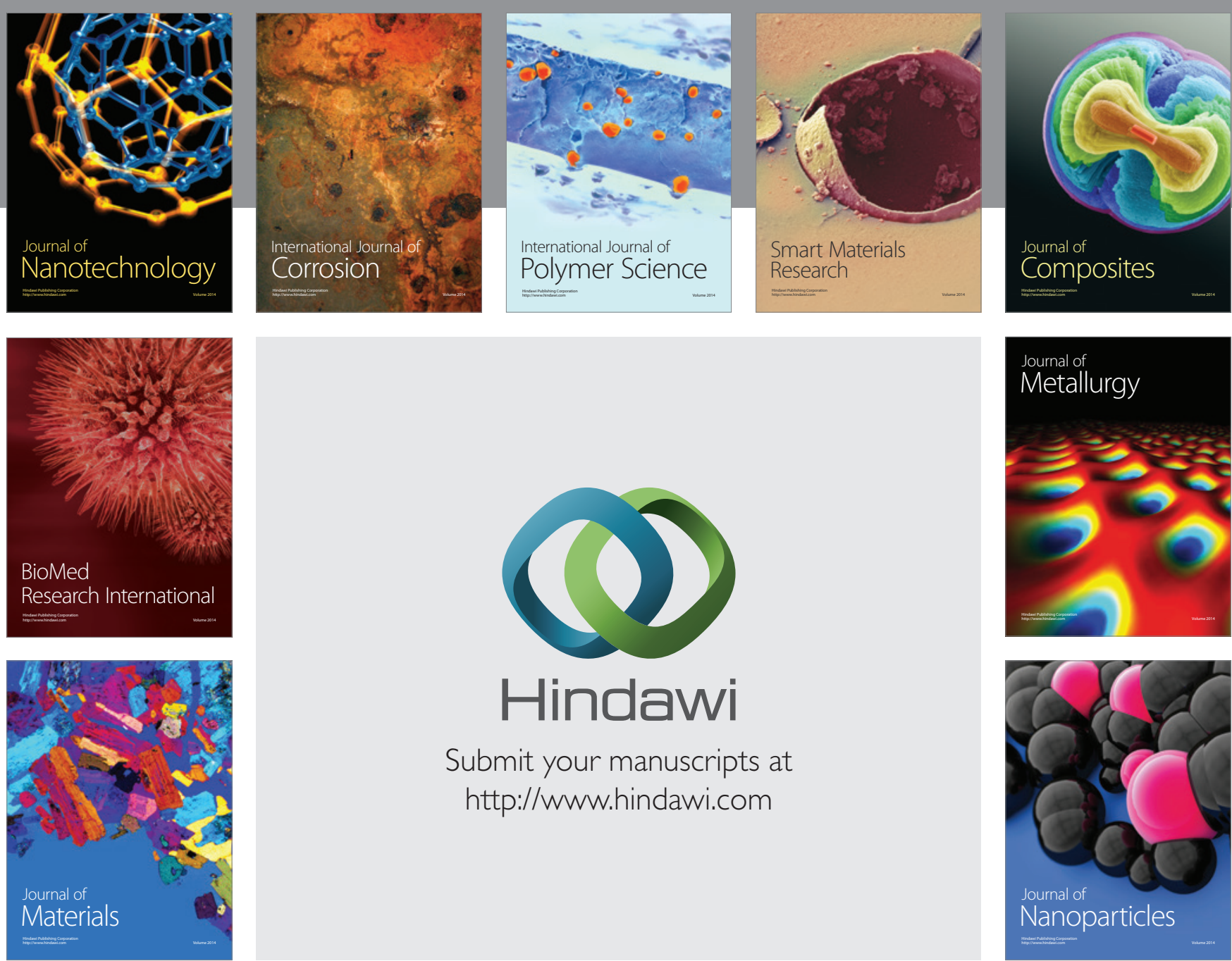

\section{Hindawi}

Submit your manuscripts at

http://www.hindawi.com

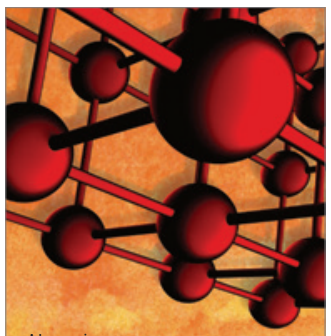

Materials Science and Engineering
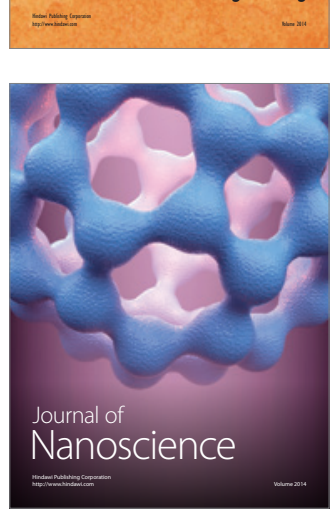
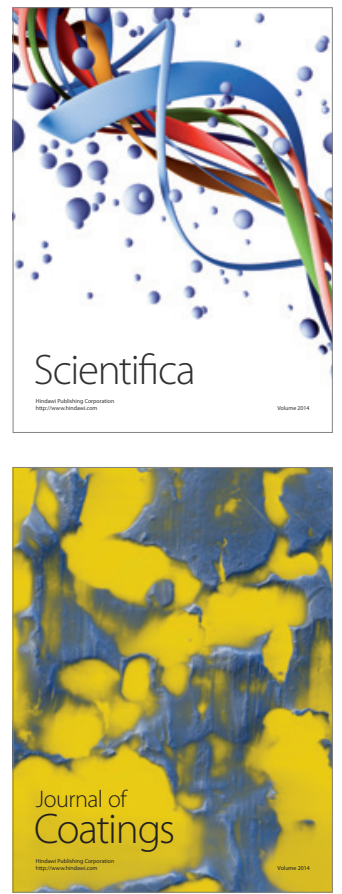
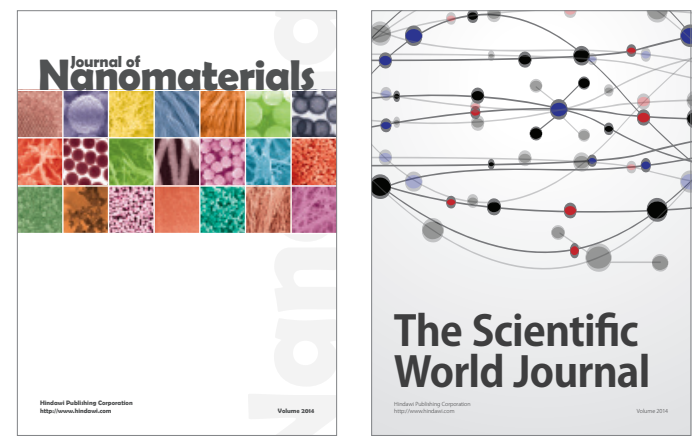

The Scientific World Journal
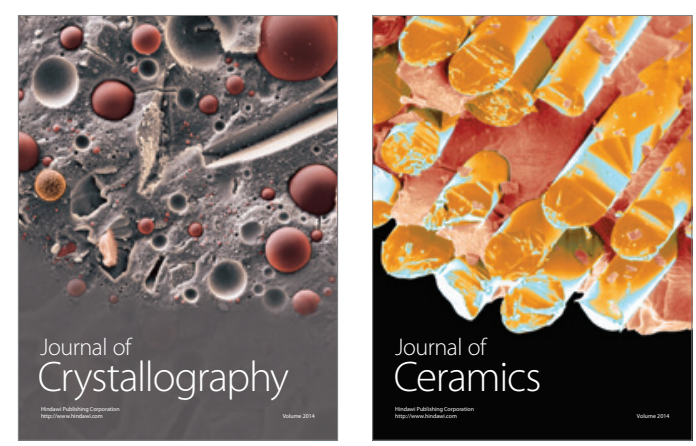
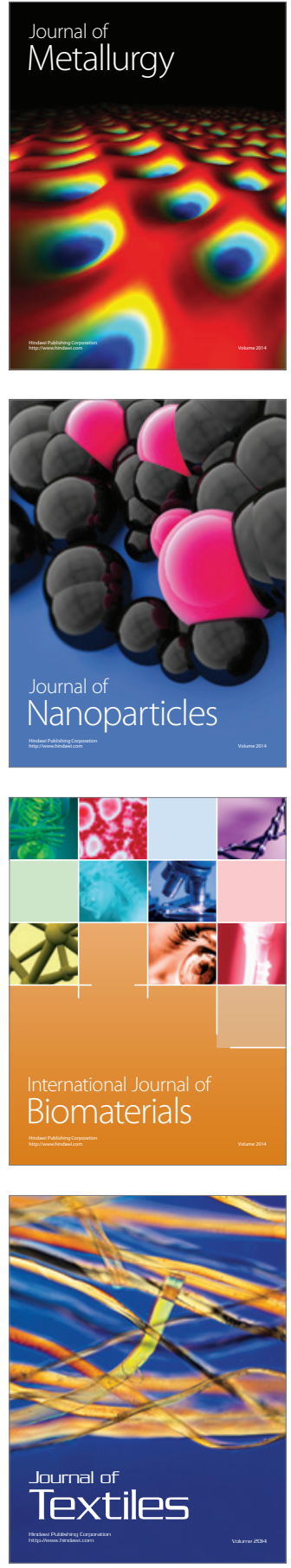